\title{
Energy Detection in Full-Duplex Cognitive Radios under Residual Self-interference
}

\author{
Taneli Riihonen and Risto Wichman \\ Department of Signal Processing and Acoustics \\ Aalto University School of Electrical Engineering \\ P.O. Box 13000, FI-00076 Aalto, Helsinki, Finland \\ \{taneli.riihonen, risto.wichman\}ealto.fi
}

\begin{abstract}
This paper investigates the possibility to exploit inband full-duplex wireless technology for simultaneous spectrum sensing and data transmission in cognitive radio terminals. The focus is especially on the receiver operating characteristics of basic energy detection. Full-duplex operation suffers from selfinterference, even after cancellation since it is imperfect in practice. The effect on the probability of missed detection is analyzed, and increasing sensing time is proposed as a countermeasure for residual distortion which, unlike in half-duplex operation, does not cause large overhead due to lost transmission opportunities. The study also compares the two- and single-antenna implementations of full-duplex radios and elicits a channel imbalance problem in the former due to which sensing provides different information than the transmitting antenna would observe.
\end{abstract}

\section{INTRODUCTION}

Cognitive radios are conventionally presumed to be halfduplex devices which implies that a secondary user (SU) in an overlay system needs to take breaks from transmission for detecting whether a primary user (PU) has become active. Alternatively, a half-duplex SU may rely on cooperation with other nearby users who are in a sensing state. Conventional cognitive radios thus suffer from inherent overhead due the half-duplex constraint: either lost transmission opportunities during sensing periods or extra channel uses and transmission energy required by feedback among cooperative users.

In-band full-duplex operation, i.e., simultaneous transmission and reception on a single channel, has been previously considered impossible in wireless systems (due to selfinterference) but the research community has now shown great determination to refute the status quo. The keys for successful full-duplex operation are efficient interference cancellation and adaptation to inevitable residual distortion. These aspects are explained well by a recent tutorial on full-duplex wireless [1]. Especially, also cognitive radio systems could benefit significantly from the ability to sense and transmit simultaneously.

Literature on full-duplex cognitive radios is still rather limited although the subject is timely and receiving increasing attention. Recognizing the potential of full-duplex operation, the main references for the present work are [2]-[14]. Especially, articles [3], [4], [7], [10], [11], [14] formulate and solve research problems related to spectrum sensing in full-duplex radios under self-interference, which is similar to the scope adopted herein. Another emerging research trend concerns cooperative relaying systems [2], [6], [8], [12], [13] when they employ full-duplex cognitive radios.

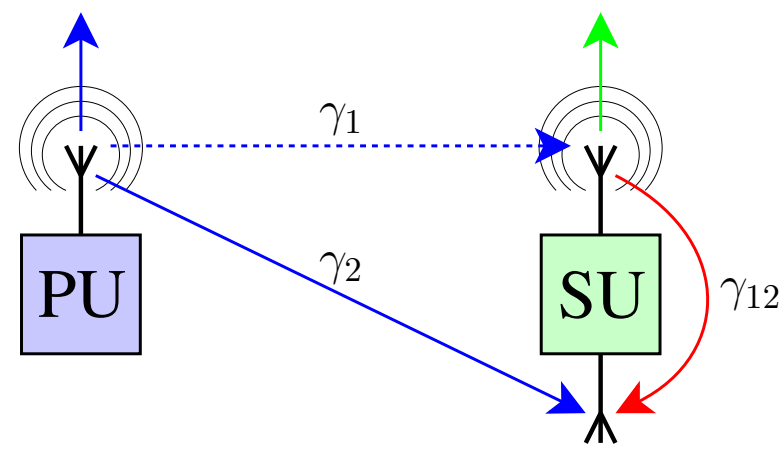

Fig. 1. A two-antenna full-duplex (FD2) secondary user (SU) exploits one antenna for sensing the potential transmission of a primary user (PU) while simultaneously transmitting to a secondary receiver from the other antenna. Variables $\gamma_{1}, \gamma_{2}$, and $\gamma_{12}$ denote instantaneous signal-to-noise ratios (SNRs).

This paper concerns energy detection for spectrum sensing in full-duplex cognitive radios in the presence of residual self-interference due to imperfect cancellation. In particular, full-duplex operation is contrasted with the reference case of switching between sensing and transmitting in conventional half-duplex cognitive radios. Hence, the first research objective is to study how the additional distortion decreases the probability of detecting PU transmission in comparison to half-duplex sensing without self-interference and how the effect could be compensated by using a longer integration period.

In order to relieve the difficulty of self-interference cancellation by improving physical isolation, full-duplex transceivers will be most likely implemented with two antennas as illustrated in Fig. 1, where the SU uses the upper antenna for transmitting and the lower antenna for sensing. This creates a gain imbalance problem which is investigated as the second research objective: A full-duplex SU aims to detect PU transmission through a different channel than a half-duplex SU.

The third research objective is to compare a two-antenna full-duplex SU with its alternative single-antenna implementation where the same antenna is used simultaneously for sensing and transmitting. In particular, a single-antenna SU is probably subject to pronounced residual self-interference but the signal from the PU propagates through the same channel as with the half-duplex counterpart. It should be noted that the SU is assumed to have only a single transceiver throughout the paper. 


\section{SySTEM MODELS}

The overall system setup during simultaneous sensing and transmission is sketched in Fig. 1 assuming a two-antenna secondary user (SU). Figure 2 illustrates more specifically the three SU variations considered in this study. The SU is assumed always to use the same antenna element for transmission while a full-duplex radio may exploit an extra antenna element for sensing; the number of transmitter/receiver pairs is still one and additional electrical switches may be thus required for facilitating two-way communication.

The following signal models presume that a primary user (PU) is active and transmitting an unknown deterministic signal $x(t)$ whose energy is defined as $\int_{t_{0}}^{t_{0}+T} x^{2}(t) \mathrm{d} t \triangleq E_{x}$.

Fig. 2(a): A single-antenna half-duplex (HD) transceiver is the conventional reference case. The half-duplex SU is not able to receive during transmission, while the received signal during a sensing break is given by

$$
y_{1}(t)=h_{1} x(t)+n_{1}(t)
$$

where $h_{1}$ is the channel amplitude coefficient from the PU to the SU and $n_{1}(t)$ represents additive Gaussian thermal noise whose variance is given by $\mathcal{E}\left\{n_{1}^{2}(t)\right\} \triangleq \sigma_{1}^{2}$. The receiver signal-to-noise ratio (SNR) becomes

$$
\gamma_{\mathrm{HD}}=\gamma_{1} \triangleq \frac{h_{1}^{2} E_{x}}{\sigma_{1}^{2}}
$$

Fig. 2(b): A two-antenna full-duplex (FD2) transceiver is the baseline implementation which facilitates simultaneous sensing and transmission. After self-interference cancellation (which is assumed to be imperfect due to residual distortion), the received signal at the sensing antenna can be modeled as

$$
y_{2}(t)=h_{2} x(t)+d_{12}(t)+n_{2}(t)
$$

where $h_{2}$ is the channel amplitude coefficient from the PU to the sensing antenna, $d_{12}(t)$ represents residual distortion, and $n_{2}(t)$ is additive Gaussian thermal noise with variance $\mathcal{E}\left\{n_{2}^{2}(t)\right\} \triangleq \sigma_{2}^{2}$. Furthermore, $\mathcal{E}\left\{d_{12}^{2}(t)\right\} \triangleq \gamma_{12} \sigma_{2}^{2}$ where parameter $\gamma_{12}$ specifies the level of residual distortion noise relatively to the thermal noise level, i.e., it is the effective interference-to-noise ratio (INR) after cancellation. By denoting $\gamma_{2} \triangleq h_{2}^{2} E_{x} / \sigma_{2}^{2}$, the receiver SNR can be expressed as

$$
\gamma_{\mathrm{FD} 2}=\frac{\gamma_{2}}{\gamma_{12}+1}
$$

Fig. 2(c): A single-antenna full-duplex (FD1) transceiver uses a circulator for connecting its transmitter and receiver to the same antenna. The received signal can be modeled as

$$
y_{1}(t)=h_{1} x(t)+d_{11}(t)+n_{1}(t)
$$

With $\mathcal{E}\left\{d_{11}^{2}(t)\right\} \triangleq \gamma_{11} \sigma_{1}^{2}$, the effective INR after cancellation is given by $\gamma_{11}$ and the receiver SNR can be expressed as

$$
\gamma_{\mathrm{FD} 1}=\frac{\gamma_{1}}{\gamma_{11}+1}
$$

The single-antenna full-duplex SU becomes equivalent to the half-duplex SU when it is not transmitting and thus $\gamma_{11}=0$.

The physical isolation offered by the circulator is low in comparison to signal attenuation due to physical distance and obstacles between separated antennas which makes cancellation more challenging. Thus, one may presume that $\gamma_{11} \gg \gamma_{12}$.
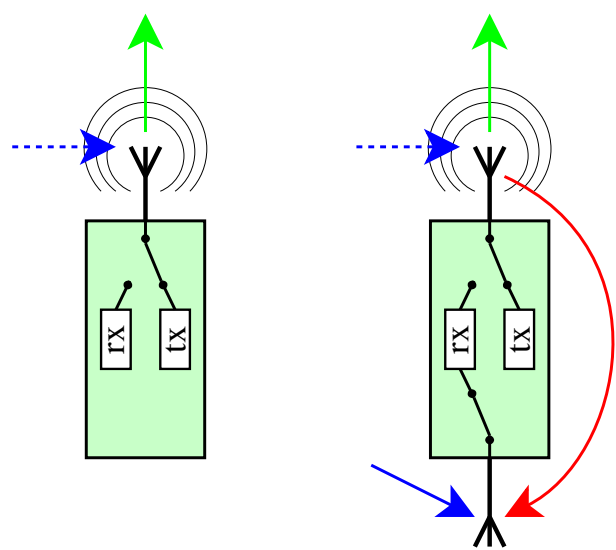

(b) FD2

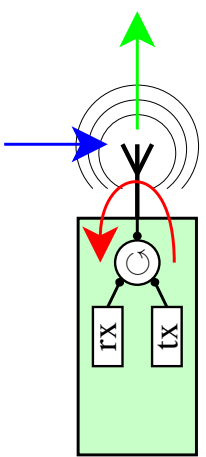

(c) FD1
Fig. 2. Three variations for implementing a cognitive radio using a single transmitter/receiver (tx/rx) pair and an optional auxiliary antenna element for facilitating full-duplex operation.

\section{ENERGY DETECTION UNDER RESIDUAL SELF-INTERFERENCE}

The secondary user aims at sensing whether an unknown deterministic bandpass signal $x(t)$ is being transmitted by a primary user; its bandwidth is $W$. This is challenging during full-duplex operation because the detection process is subject to residual distortion noise $d(t)$ due to imperfect interference cancellation in addition to usual thermal noise $n(t)$.

Letting $H_{0}$ and $H_{1}$ refer to the respective hypotheses of signal absence and presence, the received signal can be modeled as

$$
y(t)= \begin{cases}d(t)+n(t), & H_{0} \\ d(t)+n(t)+h x(t), & H_{1}\end{cases}
$$

where $h$ denotes a channel amplitude coefficient from the transmit antenna to the sensing antenna. The signal-to-noise ratio (SNR) is defined as $\gamma \triangleq h^{2} E_{x} / N_{0}$, where $E_{x}$ denotes the signal energy and the power spectral density of distortion noise plus thermal noise is given by $N_{0}$.

The energy detector integrates the received signal after filtering and squaring over a period of $T$ which yields a decision variable as $Y=\left(2 / N_{0}\right) \int_{t_{0}}^{t_{0}+T} y^{2}(t) \mathrm{d} t$. The value of $Y$ is then compared to a pre-defined energy threshold $\lambda$ such that signal presence is concluded whenever $Y>\lambda$. Assuming Gaussian distortion and noise, $Y$ follows central (resp. noncentral) chi-square distribution under $H_{0}$ (resp. $H_{1}$ ) [15].

As shown in [16], the probability of false alarm and the probability of missed detection are respectively given by

$$
\begin{aligned}
& P_{\mathrm{f}} \quad=\operatorname{Pr}\left(Y>\lambda \mid H_{0}\right)=\frac{\Gamma\left(u, \frac{\lambda}{2}\right)}{\Gamma(u)} \\
& P_{\mathrm{m}}(\gamma)=\operatorname{Pr}\left(Y \leq \lambda \mid H_{1}\right)=1-Q_{u}(\sqrt{2 \gamma}, \sqrt{\lambda})
\end{aligned}
$$

which involve the upper incomplete gamma function $\Gamma(a, x)=\int_{x}^{\infty} t^{a-1} \exp (-t) \mathrm{d} t$, the gamma function $\Gamma(a)=$ $\Gamma(a, 0)$, the generalized Marcum-Q function $Q_{m}(a, b)=$ $\left(1 / a^{m-1}\right) \int_{b}^{\infty} x^{m} I_{m-1}(a x) \exp \left(-\left(x^{2}+a^{2}\right) / 2\right) \mathrm{d} x$, and the $n$ th-order modified Bessel function of the first kind $I_{n}(x)=$ $(1 / \pi) \int_{0}^{\pi} \cos (n \theta) \exp (x \cos \theta) \mathrm{d} \theta$. Furthermore, $u=T \cdot W$. 
When setting constant values for the decision threshold $\lambda$ and for the sensing time-bandwidth product $u$, the probability of false alarm $P_{\mathrm{f}}$ is the same for all the considered SU variations, irrespective of whether the full-duplex SU transmits during sensing or not. Especially, the level of residual distortion or the channel gains do not directly affect $P_{\mathrm{f}}$. However, it should be noted that $\lambda$ is defined relatively to the power spectral density of sensing noise which itself is higher when the full-duplex SU is subject to self-interference.

1) When the $S U$ is not transmitting, the performance of fullduplex and half-duplex operation becomes rather comparable due to the absence of self-interference in both. Depending on the adopted operation strategy, this case may correspond to the situation after the SU has detected (correctly or not) the presence of an active PU and backed off from transmitting to avoid a collision, or a half-duplex SU takes a sensing break.

The probability of missed detection is $P_{\mathrm{m}}\left(\gamma_{2}\right)$ for a twoantenna full-duplex (FD2) secondary user. In comparison, both a half-duplex (HD) user and a single-antenna full-duplex (FD1) user miss detection with a different probability of $P_{\mathrm{m}}\left(\gamma_{1}\right)$ which highlights the channel gain imbalance problem. However, when a FD2 user is not transmitting, exploiting an extra electrical switch could render all the variations equivalent by connecting receivers to the same antenna in all of them.

2) When the $S U$ is transmitting, the three transceiver variations perform rather differently. Especially, the two- and singleantenna full-duplex radios sense PU presence through different channels under self-interference. This case may correspond to the situation after the SU has detected (correctly or not) that the channel is vacant and consequently launched its own transmission, or the adopted operation strategy does not allow a sensing break for a half-duplex SU.

The probability of missed detection is $P_{\mathrm{m}}\left(\gamma_{\mathrm{FD} 2}\right)$ given (4) and $P_{\mathrm{m}}\left(\gamma_{\mathrm{FD} 1}\right)$ given (6) for two- and single-antenna users, respectively. A HD user cannot sense while transmitting and, thus, it inevitably misses detecting potential PU transmission, i.e., $P_{\mathrm{m}}=1$. However, it is worth to note for reference that the HD user would miss detection in the same situation with a probability of $P_{\mathrm{m}}\left(\gamma_{\mathrm{HD}}\right)$ given (2), if it was sensing instead of transmitting. In general, it is reasonable to expect that $P_{\mathrm{m}}\left(\gamma_{\mathrm{HD}}\right)<P_{\mathrm{m}}\left(\gamma_{\mathrm{FD} 2}\right)<P_{\mathrm{m}}\left(\gamma_{\mathrm{FD} 1}\right)$, when $u$ is a constant, due to residual self-interference distortion in full-duplex sensing.

\section{NumericAl RESUlts AND Discussion}

Figure 3 illustrates the complementary receiver operating characteristics (ROC) which are computed using (8) as described above. In Fig. 3(b), the secondary user is transmitting and, thus, trivially $\left(P_{\mathrm{f}}, P_{\mathrm{m}}\right)=(?, 1)$ in the case of halfduplex (HD) operation, i.e., the probability of false alarm is indeterminate while sensing is impossible.

First of all, the ROC curves highlight the significant impact of residual self-interference in simultaneous full-duplex transmission and sensing: The probability of false alarm and the probability of missed detection are both increased by more than one magnitude when the SU begins to transmit during sensing. The performance of a single-antenna SU is worse than that of a two-antenna SU due to higher residual distortion, but it is equal to that of a half-duplex SU when only sensing.

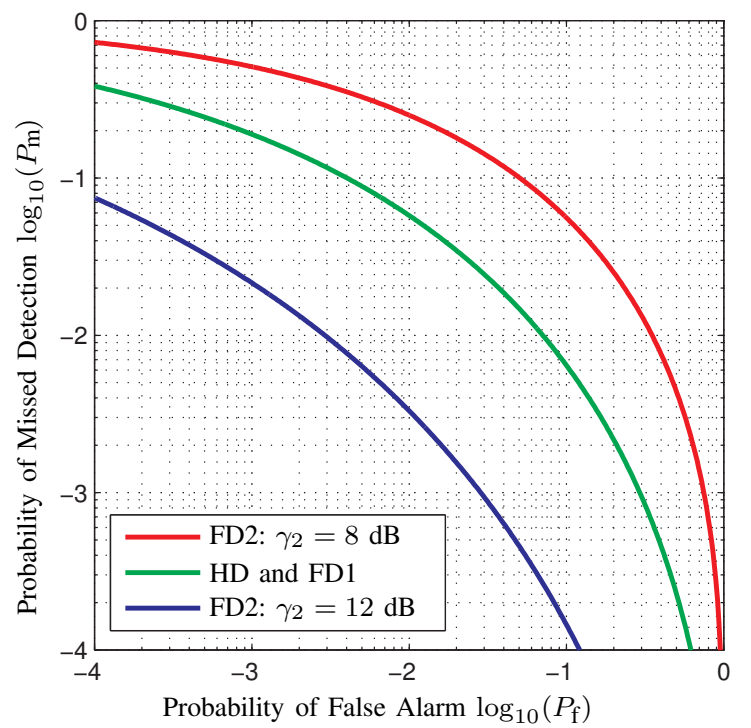

(a) SU is only sensing

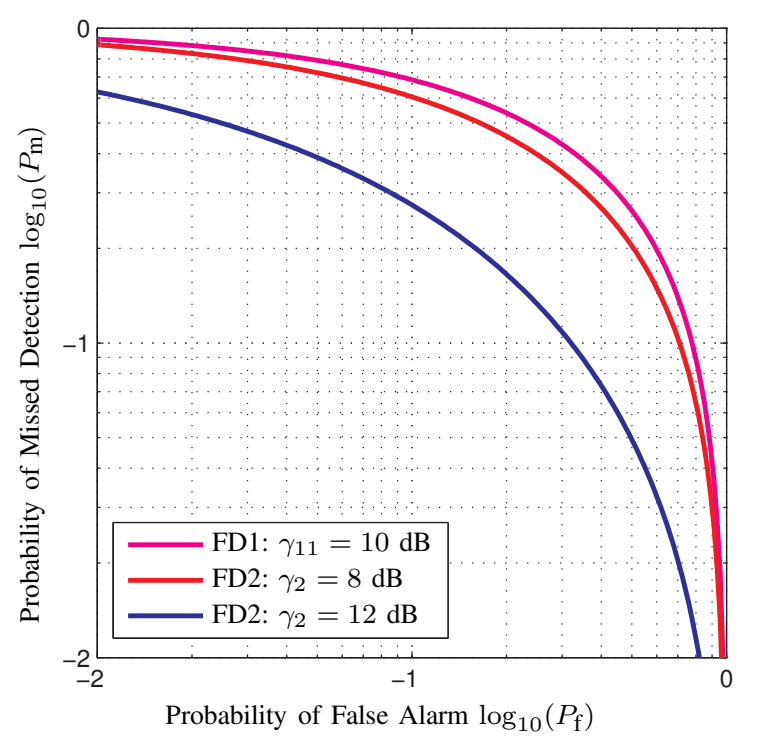

(b) SU is simultaneously transmitting and sensing

Fig. 3. Complementary ROC curves when $\gamma_{1}=10 \mathrm{~dB}, \gamma_{2} \in\{8,12\} \mathrm{dB}$, $\gamma_{12}=6 \mathrm{~dB}, \gamma_{11}=10 \mathrm{~dB}$, and $u=1$ for both half-duplex and full-duplex sensing. A half-duplex SU misses detection altogether when it is transmitting.

Figures 4 and 5 illustrate the probability of missed detection $P_{\mathrm{m}}$ in simultaneous full-duplex transmission and sensing when the probability of false alarm is fixed at $P_{\mathrm{f}}=2 \%$. For reference, a half-duplex secondary user would achieve $P_{\mathrm{m}}=3.5 \%$ in the same scenario if it was only sensing. The system parameter values are similar to those of Fig. 3.

In particular, the level of residual self-interference is varied in Fig. 4. Looking at the two extremes, full-duplex sensing could be considered ideal if the INR $\left(\gamma_{12}\right.$ or $\left.\gamma_{11}\right)$ was suppressed below $-20 \mathrm{~dB}$, while detection is almost impossible if the INR is above $15 \mathrm{~dB}$ or so. Performance is equivalent to that of half-duplex sensing in the former case except for the channel gain imbalance problem of a two-antenna SU. However, it is not realistic to assume that residual distortion is below noise. 


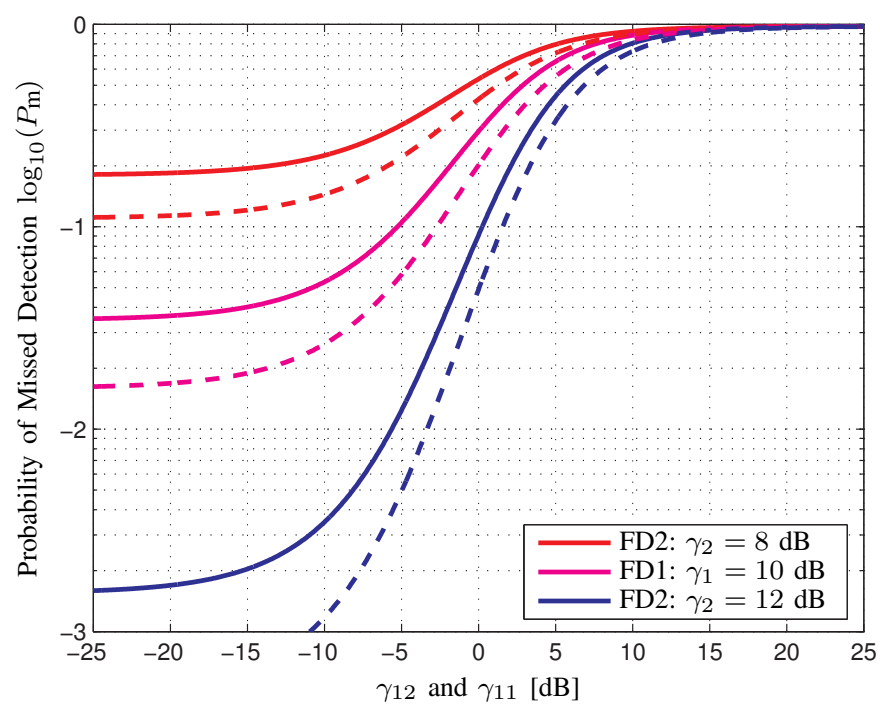

Fig. 4. Detection performance in terms of the residual self-interference levels when $P_{\mathrm{f}}=2 \%, \gamma_{1}=10 \mathrm{~dB}, \gamma_{2} \in\{8,12\} \mathrm{dB}$, and $u=1$. The dashed curves represent the corresponding cases when the sensing time is doubled.

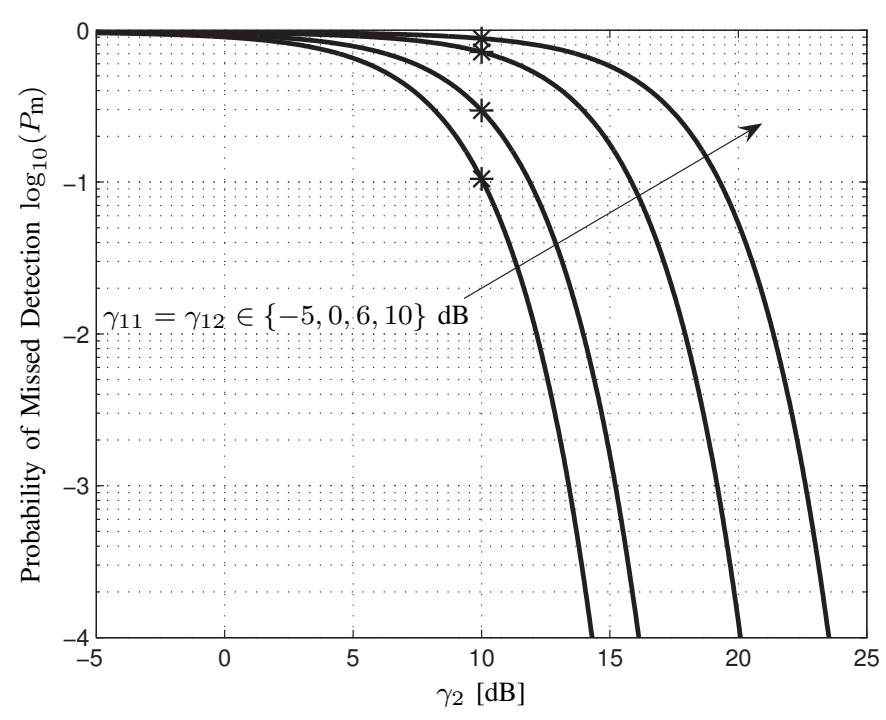

Fig. 5. Detection performance in terms of channel gain imbalance when $P_{\mathrm{f}}=2 \%, \gamma_{1}=10 \mathrm{~dB}, \gamma_{11}=\gamma_{12} \in\{-5,0,6,10\} \mathrm{dB}$, and $u=1$. The curves (resp. markers) correspond to a FD2 (resp. FD1) secondary user.

Figure 4 also illustrates how the effect of residual selfinterference could be compensated by using a longer integration period in energy detection; the sensing time is doubled in this specific example. A FD secondary user can reach the same performance as a HD user in the same scenario even in the presence of self-interference. The main constraint for increasing significantly the sensing time is the assumption that the state of a PU remains constant during the whole period.

Figure 5 illustrates the channel gain imbalance problem due to which a two-antenna full-duplex (FD2) secondary user performs differently from its single-antenna counterpart. Actually, a fortunate FD2 radio may even be able to detect PU transmission with a higher probability, and the gain imbalance can also effectively attenuate residual self-interference.

\section{CONCLUSION}

This paper studied energy detection for spectrum sensing in full-duplex cognitive radios. By performing simultaneous sensing and transmission, such radios could avoid the overhead of conventional half-duplex sensing at the cost of residual selfinterference after imperfect cancellation whose effect can be reduced by using a longer sensing period. Two full-duplex implementations were compared to conventional half-duplex sensing. The choice of an implementation was shown to be a trade-off between having a higher residual distortion level (a radio with a single antenna) or adapting to a channel gain imbalance problem (a radio with two antennas).

\section{REFERENCES}

[1] A. Sabharwal, P. Schniter, D. Guo, D. W. Bliss, S. Rangarajan, and R. Wichman, "In-band full-duplex wireless: Challenges and opportunities," IEEE Journal on Selected Areas in Communications, to appear, 2014.

[2] N. Choi, M. Patel, and S. Venkatesan, "A full duplex multi-channel MAC protocol for multi-hop cognitive radio networks," in Proc. 1st International Conference on Cognitive Radio Oriented Wireless Networks and Communications, June 2006.

[3] W. Cheng, X. Zhang, and H. Zhang, "Imperfect full duplex spectrum sensing in cognitive radio networks," in Proc. 3rd ACM Workshop on Cognitive Radio Networks, September 2011.

[4] W. Cheng, X. Zhang, and H. Zhang, "Full duplex spectrum sensing in non-time-slotted cognitive radio networks," in Proc. Military Communications Conference, November 2011.

[5] L. Ma, W. Liu, and A. Zeira, "Making overlay cognitive radios practical," in Proc. IEEE International Conference on Acoustics, Speech and Signal Processing, March 2012.

[6] H. Kim, S. Lim, H. Wang, and D. Hong, "Power allocation and outage probability analysis for secondary users in cognitive full duplex relay systems," in Proc. IEEE 13th International Workshop on Signal Processing Advances in Wireless Communications, June 2012.

[7] E. Ahmed, A. Eltawil, and A. Sabharwal, "Simultaneous transmit and sense for cognitive radios using full-duplex: A first study," in Proc. Antennas and Propagation Society International Symposium, July 2012.

[8] H. Kim, S. Lim, H. Wang, and D. Hong, "Optimal power allocation and outage analysis for cognitive full duplex relay systems," IEEE Transactions on Wireless Communications, vol. 11, no. 10, pp. 3754 3765, October 2012.

[9] H. Li, H. Lv, and H. Yuan, "Resource allocation for full duplex based cognitive radio network," in Proc. 2nd International Conference on Computer Science and Network Technology, December 2012.

[10] J. Heo, H. Ju, S. Park, and D. Hong, "Method for simultaneous sensing and data transmission in CR environments," in Proc. International Conference on Electronics, Information and Communication, JanuaryFebruary 2013.

[11] W. Afifi and M. Krunz, "Exploiting self-interference suppression for improved spectrum awareness/efficiency in cognitive radio systems," in Proc. 32nd IEEE International Conference on Computer Communications, April 2013.

[12] G. Zheng, I. Krikidis, and B. Ottersten, "Full-duplex cooperative cognitive radio with transmit imperfections," IEEE Transactions on Wireless Communications, vol. 12, no. 5, pp. 2498-2511, May 2013.

[13] W. Lu and J. Wang, "Opportunistic spectrum sharing based on fullduplex cooperative OFDM relaying," IEEE Communications Letters, vol. 18, no. 2, pp. 241-244, February 2014.

[14] J. Heo, H. Ju, S. Park, E. Kim, and D. Hong, "Simultaneous sensing and transmission in cognitive radio," IEEE Transactions on Wireless Communications, to appear, 2014.

[15] H. Urkowitz, "Energy detection of unknown deterministic signals," Proceedings of the IEEE, vol. 55, no. 4, pp. 523-531, April 1967.

[16] F. F. Digham, M.-S. Alouini, and M. K. Simon, "On the energy detection of unknown signals over fading channels," IEEE Transactions on Communications, vol. 55, no. 1, pp. 21-24, January 2007. 